\title{
Classical Quantum Mechanics - Particle is the Pulse of Matter Waves
}

\section{OPEN ACCESS}

Citation: Michopoulos D. (2020) Classical Quantum Mechanics Particle is the Pulse of Matter Waves. Open Science Journal 5(4)

Received: $23^{\text {rd }}$ March 2020

Accepted: $17^{\text {th }}$ September 2020

Published: $19^{\text {th }}$ October 2020

Copyright: (c) 2020 This is an open access article under the terms of the Creative Commons Attribution License, which permits unrestricted use, distribution, and reproduction in any medium, provided the original author and source are credited.

Funding: The author received no specific funding for this work

Competing Interests: The author has declared that no competing interests exist.

\author{
Dimosthenis Michopoulos ${ }^{1 *}$ \\ ${ }^{1}$ Hellenic Open University, Greece \\ *Corresponding author: Dimosthenis Michopoulos: std143026@ac.eap.gr or \\ dimmihop@gmail.com
}

\begin{abstract}
Since the very first development of Quantum Mechanics there are many scientists who, despite the fact that everything seems to work perfectly and the experimental results verify the theoretical predictions, do not understand what is really going on. That is why there are many different interpretations of this subject nowadays [1,2]. This paper provides a new interpretation, aiming to illustrate the fact that Quantum Mechanics is actually an extension of Classical Physics, and in contrast to other interpretations we illustrate a possible experimental verification of theoretical wording.
\end{abstract}

Keywords: Quantum Mechanics, Copenhagen Interpretation, Uncertainty Principle, Experimental Verification, Wave Function, Photon's Interpretation

\section{Introduction}

The present paper attempts to give the physical interpretation behind the theory of quantum mechanics. There are many theories today about what the theory of quantum mechanics might mean, as well as many philosophical extensions [1].

This is an open topic in physics for almost a century, when the theory of quantum mechanics was formulated.

The physical interpretation of quantum mechanics presented in this paper differs from any previous attempt to interpret quantum mechanics in the following very important point: Experimental ways of verifying this particular interpretation are suggested, so that the subject remains purely scientific, avoiding philosophical interpretations.

It also results in an approach to physical theory fully in line with ClassicalNewtonian Physics, restoring a unified approach to Physics. 


\section{Methods}

\section{The statistical interpretation of the wave function}

In 1926 Max Born attributed to the wave function the statistical interpretation which could be formulated with the following proposal: «The wave function does not represent a physically observable classical wave but a «wave of probability». The square of the absolute value of the wave function gives the probability density - that is, the probability per unit of length (or volume) to find the particle in an area of the space.»[3] Since then, every time an experiment in quantum mechanics is performed, scientists have been counting the chances of finding particles (or wave - particles) in specific quantum states.[4] The magnitude that is measured is the probability:

$$
P=\frac{N_{i}}{N}[5],[6]
$$

The experimental findings are in agreement with the theoretical prediction, but the problem is that this concept makes no sense.

\section{The uncertainty principle}

The uncertainty principle of Werner Heisenberg formulated mathematically as follows:

$$
\Delta \mathrm{x} \cdot \Delta \mathrm{p} \approx \mathrm{h}
$$

It can be seen as:

$$
\Delta \mathrm{x} \cdot \Delta \mathrm{p}=\frac{\hbar}{2}
$$

or even as:

$$
\Delta \mathrm{x} \cdot \Delta \mathrm{p} \geq \frac{\hbar}{2}
$$

Such deviations could be attributed to the geometry of the vision.

In any case and beyond the strict experimental precision, for which experimental physicists deserve to speak, the uncertainty principle can be encoded in the expression:

$$
\Delta \mathrm{x} \cdot \Delta \mathrm{p}=\mathrm{h}
$$

The famous uncertainty principle is nothing different from the De Broglie's wavelength formula: 


$$
\lambda=\frac{\mathrm{h}}{\mathrm{p}} \Leftrightarrow \lambda \cdot \mathrm{p}=\mathrm{h}
$$

In terms of physical magnitudes, the uncertainty principle tells us nothing more than De Broglie's formula tells us:

Length $\mathrm{x}$ Momentum $=$ Planck's constant.

The proof of the uncertainty principle considers it to be known and uses De Broglie's formula. So it uses what it will prove. Therefore, it works tautologically!

Let's take the mathematical proof of the uncertainty principle. It is derives from the generalized formula of the uncertainty of linear algebra:

$$
(\Delta \mathrm{A})(\Delta \mathrm{B}) \geqq \frac{1}{2}|<[\mathrm{A}, \mathrm{B}]>|
$$

Knowing that:

$$
\left[\mathrm{x}, \mathrm{P}_{\mathrm{x}}\right]=\mathrm{i} \hbar
$$

we conclude that:

$$
(\Delta \mathrm{x})\left(\Delta \mathrm{P}_{\mathrm{x}}\right) \geqq \frac{\hbar}{2}
$$

But how did we get that $\left[\mathrm{x}, \mathrm{P}_{\mathrm{x}}\right]=\mathrm{i} \hbar$ ?

The operators $\mathrm{x}$ and $\mathrm{P}_{\mathrm{x}}$ result from their action on the wave function of the flat wave material

$$
\Psi(\mathrm{x}, \mathrm{t})=\mathrm{e}^{\mathrm{i}(\mathrm{kx}-\omega \mathrm{t})} \Leftrightarrow \Psi(\mathrm{x}, \mathrm{t})=\mathrm{e}^{\mathrm{i}(\mathrm{px}-\mathrm{Et}) / \hbar}
$$

where De Broglie's formula is within:

$$
\mathrm{k}=\frac{\mathrm{p}}{\hbar} \Leftrightarrow \lambda=\frac{\mathrm{h}}{\mathrm{p}}
$$

in order to be written in the form of operators the Schrödinger equation.

More in detail, starting from: $\Psi(\mathrm{x}, \mathrm{t})=\mathrm{e}^{\frac{\mathrm{i}(\mathrm{px}-\mathrm{Et})}{\hbar}}$

we have got: $\frac{\partial \Psi}{\partial \mathrm{t}}=\frac{-\mathrm{iE}}{\hbar} \Psi$ and $\frac{\partial \Psi}{\partial \mathrm{t}}=\frac{\mathrm{ip}}{\hbar} \Psi$

At this point we define the operators:

$$
\hat{\mathrm{E}}=\mathrm{i} \hbar \frac{\partial}{\partial \mathrm{t}} \quad \text { and } \quad \widehat{\mathrm{p}_{\mathrm{x}}}=-\mathrm{i} \hbar \frac{\partial}{\partial \mathrm{x}}
$$


and write the Schrödinger equation:

$$
\mathrm{i} \hbar \frac{\partial \Psi}{\partial \mathrm{t}}=-\frac{\hbar^{2}}{2 \mathrm{~m}} \frac{\partial^{2} \Psi}{\partial \mathrm{x}^{2}}
$$

in the form:

$$
\hat{\mathrm{E}} \Psi=\frac{\widehat{\mathrm{p}^{2}}}{2 \mathrm{~m}} \Psi[7]
$$

Each wave function can be written as a suitable overlapping of the flat wave material. These operators are thus created to act on waveforms containing the De Broglie formula.

If we consider that we do not know the formula of De Broglie and eliminate it from the expressions of the wave functions these operators do not work.

So what we do is to pass De Broglie's formula through a labyrinthial statistical approach and to re-emerge it in the end like a new law, with just a new name.

The uncertainty principle is this very De Broglie's formula, which is the physical law.

\section{Tunneling}

The tunnel effect is perhaps, along with the double-slit experiment, the most striking phenomenon of Quantum Mechanics

Even more impressive is that in the tunnel phenomenon, under the Born interpretation, it is not clear if the Law of Conservation of Energy is violated or not.

Quantum physics under the Born interpretation predicts the possibility of particle formation in an area banned for energy. It is wrong to say that the area is forbidden only classically. The area is forbidden energetically.

Its prediction is actually verified.

The Law of Conservation of Energy also applies to quantum mechanics. In quantum mechanics, as in classical physics, energy is conserved as a result of homogeneity of time [8].

All the explanations provided by the literature have only interpreting character without any kind of experimental verification.

Some say that the tunnel effect is interpreted by the uncertainty principle of energy time $\Delta \mathrm{E} \cdot \Delta \mathrm{t}=\mathrm{h}$, and they assume that inside the packet of electrons there are some of them which have enough energy to cross the barrier [9].

Our opinion is that if someone wants to examine what happens, in this specific scientific area, has to make experiments in the single-electron tunneling phenomenon and examine exactly the energy of the electrons which one by one passes the barrier before and after the barrier and not just to give interpreting explanations.

The good news is that this knowledge and technology exists and is very advanced and it is experimentally verified that every electron passing through the barrier has always higher energy than that. [10],[11]. 


\section{The Photon}

For electromagnetic radiation, we know well from the historical work of Scottish Theoretical Physicist James Clerk Maxwell (1831-1879) that it propagates as a wave presenting all those characteristic properties of waves (diffraction, contribution, etc).

In December 1900 Max Planck expressed the idea that energy can be transferred from radiation to matter only by packages of a certain magnitude given by the relation $\mathrm{E}=\mathrm{hf}$. At this point Albert Einstein hastened to formulate his own approach that electromagnetic radiation is not only a wave but also a particle. This particle called the photon. Einstein interpreted various phenomena with the idea of the photon such as ionization, the photoelectric phenomenon and others.

Planck never accepted the idea of Einstein's photon claiming that the photon is essentially the energy transferred from radiation to matter and not a particle. This action naturally displays quantization but has no particle character.

The evolution of the historical facts in Physics has given weight to Einstein's idea of being indifferent to Planck's beliefs.

Einstein was therefore the pioneer of quantum mechanics, in the form we know it today, introducing the idea of the double (wave and particle) character of the nature in the sense it gave to the photon, although it then opposed the statistical interpretation that faculty Copenhagen gave to quantum mechanics, advancing his own idea.

Max Planck's view is by no means to be dismissed. There is no scientific argument that rejects the Planck optics.

Electromagnetic radiation, being a wave, can be considered as the continuous succession of electromagnetic pulses.

On the other hand we know about the energy levels of atoms from their emission and radiation spectra, but also from the solution of the Schrödinger equation.

Therefore, the theory is correct that when electromagnetic radiation interacts with matter, it is its pulses that collide one after the other with the atoms. If the impact pulse has the energy to excite or ionize the atom, does it, if not the next pulse collides and so on.

$$
E=h f
$$

is the energy of the electromagnetic impact pulse that we can call photon, avoiding mentally jumps about light's dual nature and the like.

The photon is the pulse of electromagnetic radiation.

There is no scientific argument or experimental finding that forbids us from this view and it was a matter of choice to cultivate the Einstein proposal and abandon the Planck proposal.

Hence there is a logical bridging of the seemingly contradictory concepts of particle and wave in terms of light, considering that the photon is nothing more than the electromagnetic pulse which is being perceived by its interaction with matter which is having quantized energy levels as we know.

Besides, when we study the light, we must study it when interacting with matter. Another possibility, one can easily understand, we do not have. 
Thus, the types $\mathrm{E}=\mathrm{hf}$ and ${ }^{\lambda=\frac{\mathrm{h}}{\mathrm{p}}}$, which connect the "particle" sizes $\mathrm{E}$ and $\mathrm{p}$ with the "wave" sizes $\mathrm{f}$ and $\lambda$ respectively, can be interpreted as energy (E) and momentum (p) which implies the wave pulse with frequency (f) and wavelength $(\lambda)$, respectively.

Energy and momentum that can be transferred from electromagnetic radiation to matter as like energy and momentum are transferred to the elastic impact between particles, and this makes us understand the pulse of the wave as a particle.

These are the types that open the way for the development of quantum mechanics. Without them there would be no quantum mechanics, neither in the light of the statistical interpretation as we know it until today, nor in classical interpretation which we are attempting to give with this dissertation.

Proposal for experimental verification that the photon is the pulse of electromagnetic radiation.

From Electrodynamics, we know that the energy density of the electromagnetic radiation is given by the formula:

$$
\mathrm{U}_{\mathrm{EB}}=\frac{1}{2}\left(\varepsilon_{0} \mathrm{E}^{2}+\frac{1}{\mu_{0}} \mathrm{~B}^{2}\right)^{[12]}
$$

If we use monochromatic radiation (laser) and we can measure the emitted photons then the total energy of the emitted photons should be equal to the energy of the electromagnetic radiation.

We know that the energy of the photon is:

$$
\mathrm{E}_{\mathrm{ph}}=\mathrm{hf}
$$

So if we have $\mathrm{N}$ emitted photons it is found that:

$$
N \cdot h \cdot f=\iiint \frac{1}{2}\left(\varepsilon_{0} E^{2}+\frac{1}{\mu_{0}} B^{2}\right) d \tau
$$
where $d \tau$ is the elementary volume.

Experimental verification of the above type is sufficient to demonstrate that the photon is the pulse of electromagnetic radiation.

\section{Results}

\section{Matter waves}

Matter waves, the theoretical prediction of which was made by Louis De Broglie, were experimentally detected by the famous experiment of Davisson and Germer [13], where the wave behavior of the particles was confirmed and verified that for the matter waves applies the formula

$$
\lambda=\frac{\mathrm{h}}{\mathrm{p}}
$$


In particular, the wave behavior of the particles is highlighted by the double slit experiment, where even when just an electron shoots the experimental array, the known interference pattern is observed [14].

If we now extend to the matter waves the concept that we gave to the photon, that is the pulse of electromagnetic radiation, then we can say that the particle is the pulse of the matter wave.

Such a view gives logical and meaningful results to our experimental findings. The particle, as a wave pulse, diffracts and contributes to the double slit configuration and is further detected as an integer entity on our observation screen.

\section{Discussion}

\section{Transitional types}

If we consider that:

$$
v=\frac{\omega}{2 \pi}, \lambda=\frac{\mathrm{k}}{2 \pi} \quad \mathrm{k} \alpha \mathrm{l} \quad \hbar=\frac{\mathrm{h}}{2 \pi},
$$

the types we saw above, of Planck and De Broglie, are made:

$$
\omega=\frac{\mathrm{E}}{\hbar} \quad \mathrm{k} \alpha \mathrm{l} \quad \mathrm{k}=\frac{\mathrm{p}}{\hbar}
$$

physical formulas could be called transition formulas, because thanks to them becomes the transition to quantum mechanics.

\section{The Schrödinger equation}

There are several ways to approach the Schrödinger equation. The most fundamental one might be the following.

1st Way

In classical wave theory, a wave with a specified frequency and wavelength is necessarily a flat wave described by the function:

$$
\Psi(\mathrm{x}, \mathrm{t})=\mathrm{e}^{\mathrm{i}(\mathrm{kx}-\omega \mathrm{t})}
$$

Using the transition formulas, the waveform equation is:

$$
\Psi(\mathrm{x}, \mathrm{t})=\mathrm{e}^{\mathrm{i}(\mathrm{px}-\mathrm{Et}) / \hbar}
$$

By performing the following rudimentary derivations, we have:

$$
\frac{\partial^{2} \Psi}{\partial x^{2}}=-\frac{p^{2}}{\hbar^{2}} e^{\frac{i(p x-E t)}{\hbar}} \quad, \quad \frac{\partial \Psi}{\partial t}=-i \frac{E}{\hbar} e^{\frac{i(p x-E t)}{\hbar}}
$$


If we combine the above formulas with the momentum - energy classical formula

$E=\frac{p^{2}}{2 m}+V$

we have:

$$
\mathrm{i} \hbar \frac{\partial \Psi}{\partial \mathrm{t}}=-\frac{\hbar^{2}}{2 \mathrm{~m}} \frac{\partial^{2} \Psi}{\partial \mathrm{x}^{2}}+\mathrm{V} \Psi
$$

which is the well known time-dependent Schrödinger equation.

If we now follow the method of separation of variables we have:

$$
\Psi(\mathrm{x}, \mathrm{t})=\Psi(\mathrm{x}) \mathrm{f}(\mathrm{t})
$$

$$
\frac{\partial \Psi}{\partial \mathrm{t}}=\Psi \frac{\mathrm{df}}{\mathrm{dt}} \quad, \quad \frac{\partial^{2} \Psi}{\partial \mathrm{x}^{2}}=\frac{\mathrm{d}^{2} \Psi}{\mathrm{dx}^{2}} \mathrm{f}
$$

Schrodinger equation becomes:

$$
\mathrm{ih} \frac{1}{\mathrm{f}} \frac{\mathrm{df}}{\mathrm{dt}}=-\frac{\hbar^{2}}{2 \mathrm{~m}} \frac{1}{\Psi} \frac{\mathrm{d}^{2} \Psi}{\mathrm{dx}^{2}}+\mathrm{V}
$$

Now the left part of the equation is a function of $t$ and the right member a function of $\mathrm{x}$. The only case for this to happen is to equate each member of the equation with a constant for which we choose $\mathrm{E}$, as the two members have energy dimensions.

By equalizing the right-hand member of the above equation with $\mathrm{E}$ we arrive at the time-independent Schrödinger equation:

$$
-\frac{\hbar^{2}}{2 \mathrm{~m}} \frac{\mathrm{d}^{2} \Psi}{\mathrm{dx}^{2}}+\mathrm{V} \Psi=\mathrm{E} \Psi
$$

2nd Way

In the time-independent Schrödinger equation we can arrive in the following very simple way:

In Classical Physics a defined Wave Length $\lambda$ is described by the function

$$
\mathrm{u}=\mathrm{A} \sin \frac{2 \pi \mathrm{x}}{\lambda}=\mathrm{A} \sin \mathrm{kx}
$$

and satisfies the differential equation:

$$
\mathrm{u}^{\prime \prime}+\mathrm{k}^{2} \mathrm{u}=0
$$

If we use the above equation for the wave function $\Psi$ of a matter wave we have:

$$
\psi^{\prime \prime}+\mathrm{k}^{2} \Psi=0
$$

and by introducing the "ubiquitous" type of De Broglie we have:

$$
\psi^{\prime \prime}+\frac{\mathrm{p}^{2}}{\hbar^{2}} \Psi=0
$$

And importing the momentum - energy classical formula: 
$\mathrm{E}=\frac{\mathrm{p}^{2}}{2 \mathrm{~m}}+\mathrm{V}$

we again have the time-independent Schrödinger equation:

$\Psi^{\prime \prime}+\frac{2 \mathrm{~m}}{\hbar^{2}}(\mathrm{E}-\mathrm{V}(\mathrm{x})) \Psi=0$

Or

$$
-\frac{\hbar^{2}}{2 \mathrm{~m}} \frac{\mathrm{d}^{2} \Psi}{\mathrm{dx}^{2}}+\mathrm{V} \Psi=\mathrm{E} \Psi
$$

\section{The wave function $\Psi$}

The above developments, which lead in one way or another to the Schrödinger equation, exist in almost all undergraduate quantum mechanics books.

In the present study, it was presented to make it easy to see that the Schrödinger equation, which is the foundation stone of Quantum Mechanics, does not come from nowhere, but it arises by combining a series of well-known relations of Physics.

And we say this to demonstrate that the Schrödinger equation is drawn from the "innards" of Physics.

It is therefore completely foreign to Physics the suggestion that Quantum Physics says today, that wave function $\Psi$ does not correspond to physical magnitude.

It is absurd to say that this physical magnitude does not exist and that $\Psi$ is only a mathematical entity.

The $\Psi$ is a physical quantity. Within formulas, it is combined with other physical quantities. Corresponds to the fluctuation of matter. It describes the matter waves.

This demonstrates the double slit experiment. This demonstrates the experiment of Davisson and Germer.

Why the wave function $\Psi$ is not experimentally detectable

Under the present physical interpretation, that the particle is the pulse of the wave material, this question can be answered in the sense that there is nothing else to detect.

This matter fluctuation is detected as the particle. There is nothing else for us to wait to detect and rightly, our experimental devices do not detect anything else.

Mathematical Compatibility with Born's Interpretation

The only thing left to be explained is how our theory, that the particle is the pulse of the matter wave, is mathematical compatible with the experimental verified Born's rule.

As we have said before the magnitude that is measured in experiments is the probability: 


$$
P=\frac{N_{i}}{N}[5],[6]
$$

According to the classical wave theory, the total energy of a pulse is proportional to the square of wave's equation amplitude [15].

$$
E=c A^{2}
$$

We also know that:

$$
E=h f=h \frac{N}{t}
$$

where $\mathrm{N}$ is the number of particle-pulses fired from a source at time $t$.

Let's say that $\mathrm{N}_{1}$ pulses have amplitude $\mathrm{A}_{1}$

$\mathrm{N}_{2}$ pulses have amplitude $\mathrm{A}_{2}$

$\mathrm{N} \nu$ pulses have amplitude $\mathrm{A} \nu$

$$
\begin{aligned}
& \mathrm{N}_{1}+\mathrm{N}_{2}+\cdots+\mathrm{N}_{\mathrm{v}}=\mathrm{N} \\
& \text { (34),(36) } \rightarrow h \frac{N_{i}}{t}=c A_{i}^{2} \leftrightarrow N_{i}=\frac{c t}{h} A_{i}^{2}=d A_{i}^{2} \\
& \text { where } d=\frac{c t}{h} .
\end{aligned}
$$

The probability of finding a particle in a given region of space is equal to:

$$
P_{i}=\frac{N_{i}}{N}=\frac{A_{i}^{2}}{A_{1}^{2}+A_{2}^{2}+\cdots+A_{v}^{2}}
$$

Thus, the probability of finding a particle in a given region of space is related to the amplitude of the pulse and hence to its energy.

\section{Conclusions}

Although the mathematical formulas of probability and energy are related in the above way, the physical significance is completely different in each case. And to be more precise, there is no physical significance in the Copenhagen school's view, while our theory restores classical perception to physics. It is essential for each particle to have the energy needed to be found in a particular region of space, and any uncertainty in measurement is due to our inherent inability to know precisely the initial conditions, just as in classical physics.

\section{References:}

1.Interpretation of Quantum Theory, An overview, Dimitris K. Lazarou, University of Athens, Greece, Physics Department, Nuclear and Elementary Particle Physics section, https://arxiv.org/ftp/arxiv/papers/0712/0712.3466.pdf

2.Richard P Feynman: Quantum Mechanical View of Reality 1 (Part1) https://www.youtube.com/watch?v=72us6pnbEvE

3.Born, Max (11 December 1954). "The statistical interpretation of quantum mechanics" https://www.nobelprize.org/uploads/2018/06/born-lecture.pdf

4.https://www.feynmanlectures.caltech.edu/III_03.html

5.https://arxiv.org/pdf/1207.7214.pdf

6.Experiments in Modern Physics 2nd Edition Adrian C. Melissinos, Jim Napolitano, ISBN-13: $978-$ 0124898516, ISBN-10: 0124898513, Compton Scattering pg. 379-380

7.Introduction to Quantum Mechanics, David J. Griffiths, ISBN 0-13-124405-1, pg. 108-110

8.Wigner E.P. (1997) Conservation Laws in Classical and Quantum Physics. In: Wightman A.S. (eds) Part I: Particles and Fields. Part II: Foundations of Quantum Mechanics. The Scientific Papers, 
vol A / 3. Springer, Berlin, Heidelberg,DOIhttps://doi.org/10.1007/978-3-662-09203-3_11 [9] Interpretation of Electron Tunneling from Uncertainty Principle Angik Sarkar Department of Electrical Engineering,Indian Institute of Technology,Kharagpur,India

10.Single-Electron Tunneling, Gerd Schon Institut fur Theoretische Festkorperphysik, Universitat Karlsruhe, D-76128 Karlsruhe, Germany

11.Pronounced Effect of pn-Junction Dimensionality on Tunnel Switch Threshold Shape

Sapan Agarwal and Eli Yablonovitch Electrical Engineering \& Computer Sciences Dept., University of California, Berkeley, 253 Cory Hall, Berkeley, CA, 94720

12.Introduction toelectrodynamics Fourth Edition David J. Griffiths, pg.357

13.A proper understanding of the Davisson and Germer experiments for undergraduate modern physics course Masatsugu Suzuki* and Itsuko S. Suzuki† Department of Physics, State University of New York at Binghamton, Binghamton NY 13902-6000 (Dated: July 24, 2013)

14. https://physicsworld.com/a/the-double-slit-experiment/

15.Serway, Physics for Scientists and Engineers, 6th edition, pg 502. 